\title{
A case of repeat hepatectomy for liver metastasis from solid pseudopapillary neoplasm of the pancreas: a case report
}

\author{
Atsushi Morito ${ }^{1}$, Kojiro Eto ${ }^{1}$, Kozue Matsuishi ${ }^{1}$, Hirokazu Hamasaki ${ }^{1}$, Keisuke Morita' ${ }^{1}$ Satoshi Ikeshima ${ }^{1}$, \\ Kei Horino', Shinya Shimada ${ }^{1}$ and Hideo Baba ${ }^{2^{*}}$ (D)
}

\begin{abstract}
Background: Solid pseudopapillary neoplasm of the pancreas is a rare tumor in young women, metastasizing in only 5-15\% of cases, and most commonly to the liver. Although treatment guidelines have not been established, surgical resection is usually performed. We report a rare case of repeat hepatectomy for liver metastases after distal pancreatectomy with solid pseudopapillary neoplasm.

Case presentation: The patient was a 71-year-old woman who underwent distal pancreatectomy for solid pseudopapillary neoplasm, and liver metastasis occurred 4 years after the first surgery. Partial liver resection was performed for four liver metastases, and histopathological examination revealed a diagnosis of liver metastasis from solid pseudopapillary neoplasm. However, 18 months later, liver metastases were detected again; three tumors were identified, and partial resection was performed, which has provided 18 months' recurrence-free survival.
\end{abstract}

Conclusions: Long-term prognosis can be expected following Ro resection for resectable liver metastasis from solid pseudopapillary neoplasm.

Keywords: Solid pseudopapillary neoplasm, Pancreas, Liver metastasis, Repeat hepatectomy

\section{Background}

Solid pseudopapillary neoplasm (SPN) of the pancreas is a rare tumor that typically occurs in young women, accounting for only $1-2 \%$ of pancreatic tumors [1]. Since Frantz first reported SPN in 1959, the number of reported cases has increased [2]. The clinicopathologic features of SPN are unique: slow-growing, low-grade malignancy [3]. SPN metastasizes in only $5-15 \%$ of all cases, and common sites include the liver, spleen, omentum, peritoneum, duodenum [4], 5. Surgical resection is considered the most efficient treatment option for SPN. However, the management of metastatic tumors of SPN

\footnotetext{
*Correspondence: hdobaba@kumamoto-u.ac.jp

2 Department of Gastroenterological Surgery, Graduate School of Medical Sciences, Kumamoto University, 1-1-1 Honjo, Chuo-ku, Kumamoto 860-8556, Japan

Full list of author information is available at the end of the article
}

is unclear. We report a case of repeat hepatectomy for liver metastasis from SPN.

\section{Case presentation}

A 71-year-old woman underwent distal pancreatectomy without lymph node dissection for SPN of the pancreas in 2013. The histopathological diagnosis showed solid diffuse growth of circular eosinophilic tumor cells with cystic and hemophilic changes on pseudopapillary structure. In addition, it was partially necrotic and bleeding. Immunohistostaining studies revealed that the tumor cells were positive for vimentin, synaptophysin, cluster of differentiation (CD) $56, \beta$-catenin, CD10, and progesterone receptor. In 2017 (4 years from the first surgery), computed tomography $(\mathrm{CT})$ identified four nodules in the anterior and posterior segments of the right lobe of the liver measuring approximately $15 \mathrm{~mm} \times 14 \mathrm{~mm}$, 
$12 \mathrm{~mm} \times 11 \mathrm{~mm}, 8 \mathrm{~mm} \times 8 \mathrm{~mm}$, and $5 \mathrm{~mm} \times 4 \mathrm{~mm}$ (Fig. 1a, b); all had low-density areas. Abdominal magnetic resonance imaging (MRI) confirmed the four nodules and revealed hypointensity in the hepatobiliary phase (Fig. 1c-f). Routine laboratory data, including tumor markers, were within normal ranges. Because these tumors were suspected metastases from SPN according to the imaging findings, the patient underwent partial hepatectomy. Pathological examination of the tumors revealed solid growth of circular eosinophilic tumor cells with cystic and hemophilic changes (Fig. 2a, b). In addition, the tumor embolization into the portal vein was observed. Immunohistochemical studies revealed that the tumor cells were positive for vimentin, synaptophysin, CD56, $\beta$-catenin, CD10, and progesterone receptor. This was the same result as the first surgery. The Ki-67 labeling index was 3\%. These findings closely resembled the initial surgical pancreatic SPN, and we diagnosed SPN metastases. In 2018 (18 months from the first liver resection), we detected recurrent liver metastases. CT identified three low-density areas in the right liver lobe measuring approximately $10 \mathrm{~mm} \times 9 \mathrm{~mm}$, $9 \mathrm{~mm} \times 8 \mathrm{~mm}$, and $6 \mathrm{~mm} \times 6 \mathrm{~mm}$ (Fig. 3-a and b). MRI confirmed the tumors (Fig. 3c-f). Because the imaging findings were the same as the previous findings, we considered the new tumors to be liver metastasis from
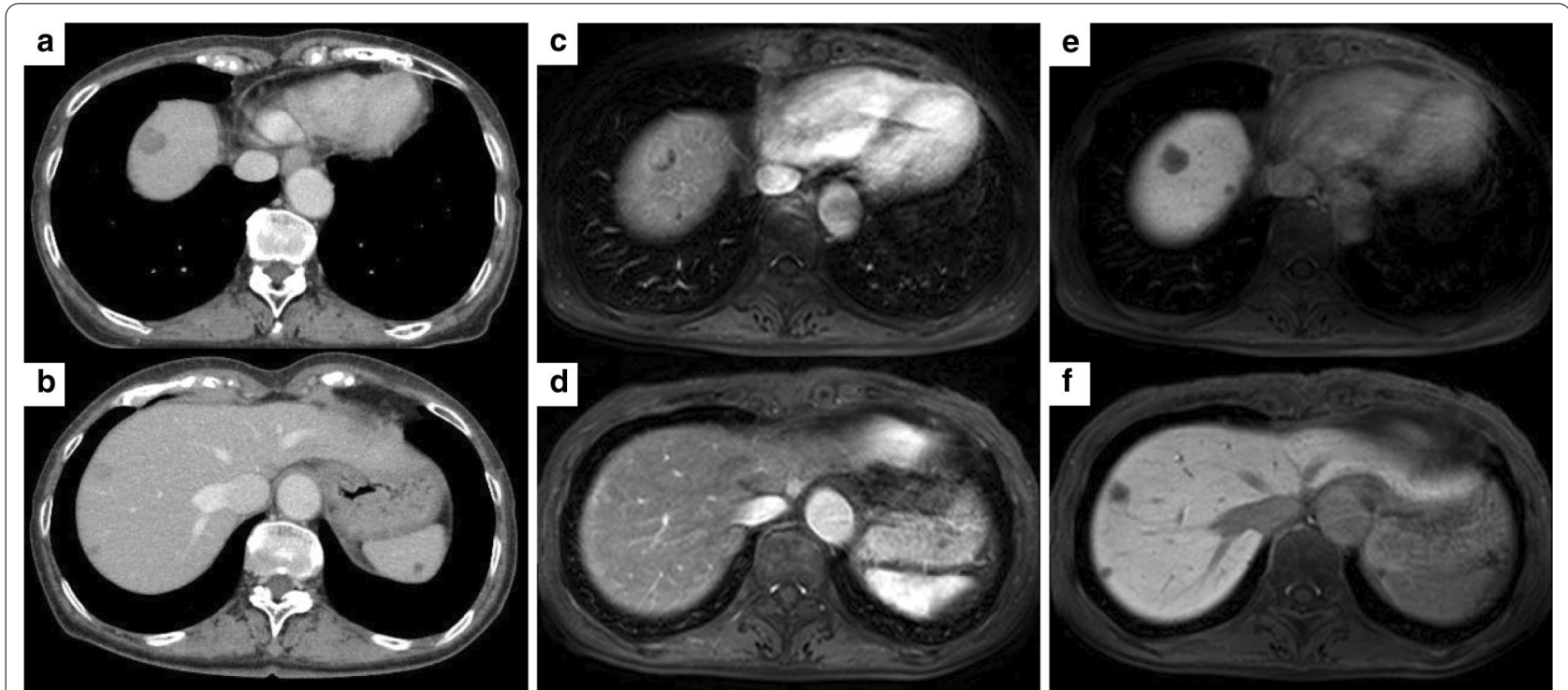

Fig. 1 a, b CT image. Four tumors are seen in liver segments S7 and S8. c-f EOB-MRI image. The tumor edges are hyperintense with dynamic imaging, and some enhancement is exhibited within the tumors

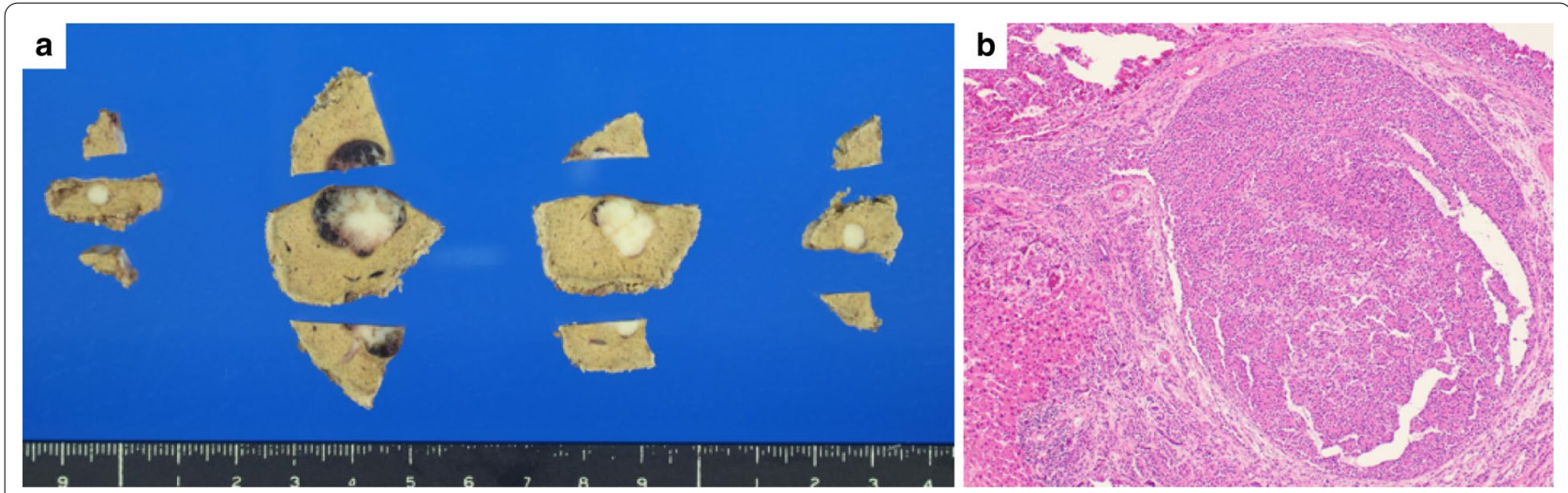

Fig. 2 Gross and histopathological findings of the excised specimens. Left image: gross tumor specimens. Right image: photomicrograph showing eosinophilic tumor cells proliferating throughout. The image shows a pseudopapillary structure centered on a thin vascular connection network. These findings are similar to the previous findings 

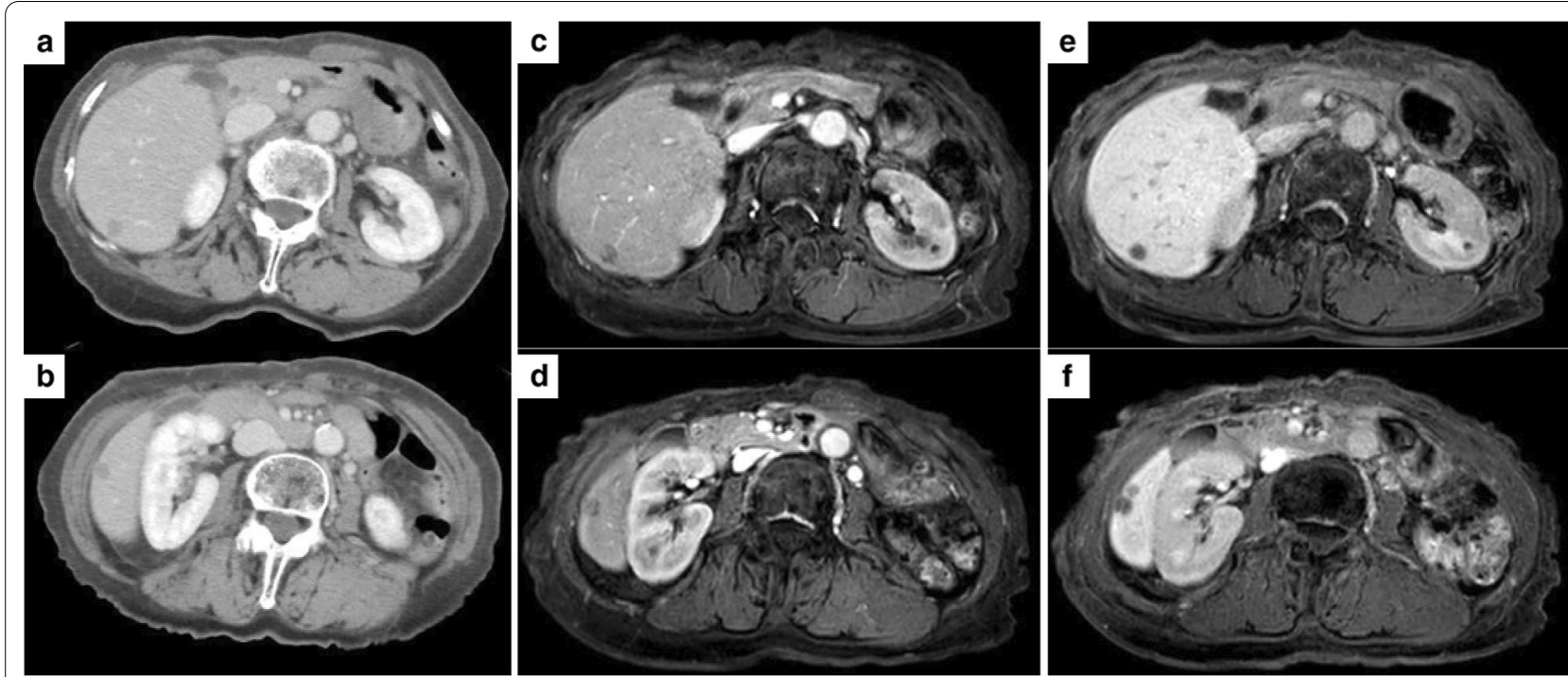

Fig. 3 a, b CT image. Three tumors are visible in liver segments S7 and S8. c-f: EOB-MRI image. The tumor edges are hyperintense with dynamic imaging, and some enhancement is exhibited within the tumors, similar to the previous findings

SPN, and the patient underwent partial re-hepatectomy. The pathological and immunohistochemical examination results were the same as those obtained previously (Fig. 4a, b). The patient has remained disease-free 18 months after the re-hepatectomy.

\section{Conclusions}

SPN of the pancreas is a rare neoplasm with low-grade malignant potential [3]. Immunopathological examination is useful for diagnosing SPN. Most pancreatic SPNs are strongly positive for CD10 (96\%), progesterone receptor $(79 \%)$, cytokeratin $(28 \%)$, synaptophysin $(26 \%)$, and chromogranin (15\%) [6]. This case was positive for CD10 and progesterone receptor; therefore, the diagnosis was liver metastasis from SPN.

Although SPN has low malignant potential, $5 \%-15 \%$ of SPN patients develop metastasis [4] [5] [7]; most commonly to the liver [8]. The number of cases is small, and treatment guidelines have not been established. There are reports of the treatment of liver metastases using chemotherapy [9] [10], transarterial chemoembolization (TACE) [11] [12], and radiofrequency ablation (RFA) [13] [14]. However, these treatments are options only for unresectable cases. In resectable cases, hepatectomy
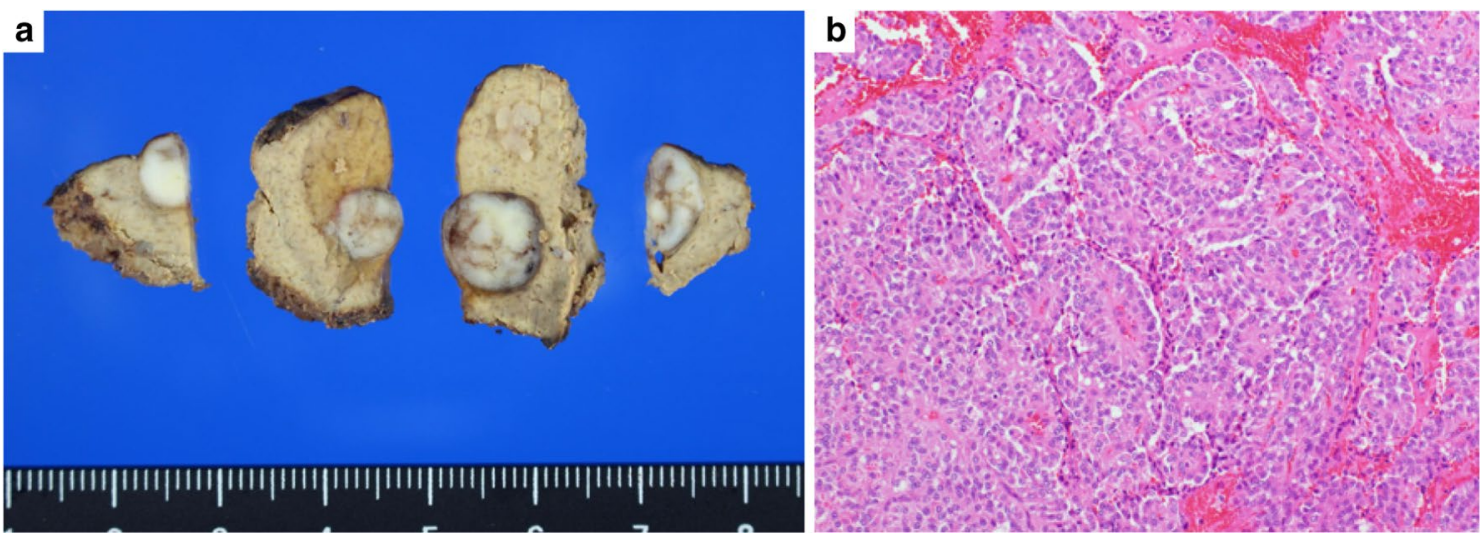

Fig. 4 Gross and histopathological findings of the excised specimens. Left image: gross tumor specimens. Right image: photomicrograph showing eosinophilic tumor cells proliferating throughout. The image shows a pseudopapillary structure centered on a thin vascular connection network. These findings are similar to the previous findings 
is considered more likely to lead a cure. In our case, the extent of resection was not wide, and liver function was maintained; hepatectomy resulted in an 18-month recurrence-free survival.

Diffuse growth, venous invasion, nuclear pleomorphism, mitotic rate, necrosis and dedifferentiation are histopathological findings suggesting high malignancy of SPN [15]. This case also showed diffuse growth and necrosis at the time of the first surgery, and that may have had a highly malignant tumor. For highly malignant SPN, resection of the metastasis site may lead to a cure.

It is necessary to select treatment considering the tumor malignancy, resection range, liver function, and the timing of the surgery.

In conclusion, we experienced a case of repeat hepatectomy for liver metastasis from SPN. 18-month recurrence-free survival was achieved by surgery.

\section{Abbreviations}

SPN: Solid pseudopapillary neoplasm; CT: Computed tomography; MRI: Magnetic resonance imaging; CD: Cluster of differentiation; TACE: Transarterial chemoembolization; RFA: Radiofrequency ablation.

\section{Acknowledgement}

We thank Jane Charbonneau, DVM, from Edanz Group (https://en-author-servi ces.edanz.com/ac) for editing a draft of this manuscript.

\section{Authors' contributions}

AM described and designed the article. KE edited the article. HB supervised the editing of the manuscript. Other remaining co-authors collected the data and discussed the content of the manuscript. All authors read and approved the final manuscript.

\section{Funding}

This study was not funded.

\section{Availability of data and materials}

All data generated or analyzed during this study are included in this published article.

\section{Ethics approval and consent to participate}

Written informed consent was obtained from the patient for publication of this case report and any accompanying images.

\section{Consent for publication}

Written informed consent was obtained from the patient for publication of this case report and any accompanying images.

\section{Competing interests}

The authors declare that they have no conflicts of interest.

\section{Author details}

${ }^{1}$ Department of Surgery, Japan Community Health Care Organization Kumamoto General Hospital, 10-10 Tori-machi, Yatsushiro, Kumamoto 866-8660, Japan. ${ }^{2}$ Department of Gastroenterological Surgery, Graduate School of Medical Sciences, Kumamoto University, 1-1-1 Honjo, Chuo-ku, Kumamoto 860-8556, Japan.
Received: 6 January 2021 Accepted: 8 February 2021

Published online: 01 March 2021

\section{References}

1. de Castro SM, Singhal D, Aronson DC, Busch OR, van Gulik TM, Obertop $H$, et al. Management of solid-pseudopapillary neoplasms of the pancreas: a comparison with standard pancreatic neoplasms. World J Surg. 2007;31(5):1130-5.

2. Lin MY, Stabile BE. Solid pseudopapillary neoplasm of the pancreas: a rare and atypically aggressive disease among male patients. Am Surg. 2010;76(10):1075-8.

3. Adams AL, Siegal GP, Jhala NC. Solid pseudopapillary tumor of the pancreas: a review of salient clinical and pathologic features. Adv Anat Pathol. 2008;15(1):39-45

4. Papavramidis T, Papavramidis S. Solid pseudopapillary tumors of the pancreas: review of 718 patients reported in English literature. J Am Coll Surg. 2005;200(6):965-72.

5. Lubezky N, Papoulas M, Lessing Y, Gistein G, Brazowski E, Nachmany I, et al. Solid pseudopapillary neoplasm of the pancreas: Management and long-term outcome. Eur J Surg Oncol. 2017;43(6):1056-60.

6. Nguyen NQ, Johns AL, Gill AJ, Ring N, Chang DK, Clarkson A, et al. Clinical and immunohistochemical features of 34 solid pseudopapillary tumors of the pancreas. J Gastroenterol Hepatol. 2011;26(2):267-74.

7. Gedaly R, Toledano A, Millan G, Essenfeld H, Zambrano VJ. Treatment of liver metastases from a solid pseudopapillary tumor of the pancreas. J Hepatobiliary Pancreat Surg. 2006;13(6):587-90.

8. Yang F, Fu DL, Jin C, Long J, Yu XJ, Xu J, et al. Clinical experiences of solid pseudopapillary tumors of the pancreas in China. J Gastroenterol Hepatol. 2008:23(12):1847-51.

9. Kang CM, Kim H, Cho Y, Kim YS, Hwang HK, Choi HJ, et al. In vitro adenosine triphosphate-based chemotherapy response assay (ATP-CRA) in solid pseudopapillary tumor of the pancreas. Pancreas. 2012;41(3):498-500.

10. Maffuz A, Bustamante Fde T, Silva JA, Torres-Vargas S. Preoperative gemcitabine for unresectable, solid pseudopapillary tumour of the pancreas. Lancet Oncol. 2005;6(3):185-6.

11. Prasad TV, Madhusudhan KS, Srivastava DN, Dash NR, Gupta AK. Transarterial chemoembolization for liver metastases from solid pseudopapillary epithelial neoplasm of pancreas: a case report. World J Radiol. 2015;7(3):61-5

12. Matsuda Y, Imai Y, Kawata S, Nishikawa M, Miyoshi S, Saito R, et al. Papillary-cystic neoplasm of the pancreas with multiple hepatic metastases: a case report. Gastroenterol Jpn. 1987;22(3):379-84.

13. Li JX, Wu H, Huang JW, Prasoon P, Zeng Y. Synchronous intraoperative radiofrequency ablation for multiple liver metastasis and resection of giant solid pseudopapillary tumors of the pancreas. Chin Med J (Engl). 2012;125(9):1661-3.

14. Foltys D, Moench C, Burck I, Hoppe-Lotichius M, Schad A, Teufel A, et al. The solid pseudopapillary tumor (SPT)-a rare neoplasm of the pancreas. Z Gastroenterol. 2008:46(7):689-94.

15. Watanabe Y, Okamoto K, Okada K, Aikawa M, Koyama I, Yamaguchi H, et al. A case of aggressive solid pseudopapillary neoplasm: Comparison of clinical and pathologic features with non-aggressive cases. Pathol Int. 2017:67(4):202-7.

\section{Publisher's Note}

Springer Nature remains neutral with regard to jurisdictional claims in published maps and institutional affiliations. 